\title{
Clinical characteristics and outcomes of spontaneous bacterial peritonitis caused by Enterobacter species versus Escherichia coli: a matched case-control study
}

Seongman Bae ${ }^{1}$, Taeeun Kim¹, Min-Chul Kim¹, Yong Pil Chong ${ }^{1}$, Sung-Han Kim¹, Heungsup Sung ${ }^{3}$, Young-Suk Lim², Sang-Oh Lee', Mi-Na Kim³, Yang Soo Kim', Jun Hee Woo ${ }^{1}$ and Sang-Ho Choi ${ }^{1 *}$

\begin{abstract}
Background: Enterobacter species are important nosocomial pathogens, and there is growing concern about their ability to develop resistance during antimicrobial therapy. However, few data are available on the clinical characteristics and outcomes of Enterobacter spontaneous bacterial peritonitis (SBP).

Methods: We retrospectively identified all patients with SBP caused by Enterobacter species admitted to a tertiary care hospital between January 1997 and December 2013. Each case was age- and sex-matched with four patients with Escherichia coli SBP.

Results: A total of 32 cases with Enterobacter SBP and 128 controls with E. coli SBP were included. Twenty-one (65.6\%) cases and 111 (86.7\%) controls had Child-Pugh class $C(P=0.006)$. Cases were significantly more likely to have hepatocellular carcinoma (65.6 \% vs. $37.5 \%, P=0.004)$ and upper gastrointestinal bleeding $(28.1 \%$ vs. $9.4 \%$, $P=0.005)$. The initial response to empirical therapy $(81.3 \%$ vs. $81.2 \%, P=0.995)$ and the 30-day mortality (37.5 \% vs. $28.9 \%, P=0.35$ ) were not significantly different between the groups. Drug resistance emerged in one case and in no controls (4.3\% [1/23] vs. $0 \%[0 / 98], P=0.19)$.
\end{abstract}

Conclusions: Compared with E. coli SBP, patients with Enterobacter SBP more frequently had hepatocellular carcinoma and upper gastrointestinal bleeding, yet clinical outcomes were comparable. Development of resistance during third-generation cephalosporin therapy was infrequent in patients with Enterobacter SBP.

Keywords: Enterobacter, Spontaneous bacterial peritonitis, Liver cirrhosis

\section{Background}

Enterobacter species are important nosocomial pathogens that cause a variety of infections, including bacteremia, pneumonia, surgical site infections, and urinary tract infections [1]. Among Enterobacteriaceae isolated from hospital-acquired infections, Enterobacter species have been ranked as the third most frequent isolate following Escherichia coli and Klebsiella species [2-7]. For more than two decades, there has been growing concern about the induction of $\beta$-lactam resistance in Enterobacter

\footnotetext{
* Correspondence: sangho@amc.seoul.kr

'Department of Infectious Diseases, Asan Medical Center, University of Ulsan College of Medicine, Seoul, Republic of Korea

Full list of author information is available at the end of the article
}

infections mediated by chromosomally encoded AmpC $\beta$ lactamase following antibiotic exposure $[8,9]$. This type of inducible resistance is reported to develop at frequencies of 8.3 to $19.6 \%$ during therapy with broad-spectrum cephalosporins for bloodstream infections $[10,11]$.

Enterobacter species are known to be part of the microbial etiology for spontaneous bacterial peritonitis (SBP), being the second to fourth most common causative organism among gram-negative bacilli [12-15]. However, there is a paucity of clinical data about the prevalence, characteristics, and outcomes of Enterobacter SBP. Although some small studies included Enterobacter species as causative organisms of SBP, the number of included episodes did not exceed five in any study and 
Enterobacter SBP has not yet been analyzed separately. Furthermore, the clinical responses and outcomes of patients treated with third-generation cephalosporins, the most widely used antimicrobials for SBP, have not yet been investigated. Therefore, with a large SBP cohort, we sought to characterize the frequency, clinical manifestations, responses to antimicrobial therapy, emergence of resistance, and outcomes of Enterobacter SBP.

\section{Methods}

\section{Study design, setting, and population}

We performed a retrospective case-matched study in Asan Medical Center, a 2700-bed tertiary care institution in Seoul, Republic of Korea. All patients over 17 years who were admitted for SBP between January 1997 and December 2013 were identified by searching for the diagnostic codes "cirrhosis" and "peritonitis" in the computerized database of our institute. Patients with secondary peritonitis, defined as peritonitis caused by perforation or inflammation of intra-abdominal organs, were excluded [16]. Patients who were receiving peritoneal dialysis were also excluded. Finally, we confirmed SBP cases through a detailed review of the medical records. All patients who had Enterobacter SBP were included in the study as cases, and they were matched with four-times as many patients with $E$. coli SBP with a similar age ( \pm 5 years) and the same sex. If more than four control patients were available for each case, we chose those patients whose dates of admission were closest to that of the case. This study was approved by the institutional review board of the Asan Medical Center.

\section{Clinical data collection and analysis}

We collected the following clinical data for each case and control: age, sex, site of acquisition of the infection, etiology of cirrhosis, comorbid diseases, Child-Pugh score, model for end-stage liver disease (MELD) score, invasive procedures performed within 30 days prior to SBP, antecedent administration of antimicrobials within 30 days, history of past hospital stays before the onset of SBP, initial clinical manifestations, laboratory findings, antimicrobial susceptibility, antibiotic therapy, and treatment outcomes.

In both cases and controls, SBP was subcategorized as either definite or probable. Definite SBP was defined by an ascitic polymorphonuclear cell (PMN) count $>250$ cells $/ \mathrm{mm}^{3}$ and a positive ascitic culture, while probable SBP was defined by an ascitic PMN count $>250$ cells/ $\mathrm{mm}^{3}$ with only a positive blood culture without any other primary focus [17].

\section{Microbiology}

Ascitic fluid samples were inoculated onto blood agar plates, MacConkey agar plates, and Brucella agar plates, and/or the BACTEC system (Becton Dickinson, Heidelberg, Germany). Identification of Enterobacter species and antimicrobial susceptibility testing were performed using the Microscan system (Dade Behring, Deerfield, IL, USA). Susceptibility to third-generation cephalosporins was defined according to the revised 2009 guidelines of the Clinical and Laboratory Standards Institute (susceptible, $\leq 8 \mu \mathrm{g} / \mathrm{ml}$; intermediate, $16-32 \mu \mathrm{g} /$ $\mathrm{ml}$; and resistant, $\geq 64 \mu \mathrm{g} / \mathrm{ml}$ ) [18].

\section{Statistical analyses}

Continuous variables were compared using Student's $t$ test and the Mann-Whitney $U$-test, while categorical variables were tested by $x^{2}$ or Fisher's exact test. To identify clinical factors associated with Enterobacter rather than E. coli SBP, univariate and multivariate analyses were conducted using a logistic regression model. The clinical variables favoring Enterobacter SBP over $E$. coli $\mathrm{SBP}$ in the univariate analysis $(P<0.1)$ were included in the multivariate analysis. All $P$ values are two-tailed, and $P<0.05$ was considered statistically significant. SPSS Statistics, version 19.0 (IBM, Armonk, NY, USA), was used for analyses.

\section{Results \\ Demographic characteristics of SBP patients}

During the 17 year period of study, a total of 1,172 episodes of culture-proven SBP were identified. Enterobacter SBP occurred in 32 patients $(2.7 \%)$, including 26 with $E$. cloacae SBP and six with $E$. aerogenes SBP. Enterobacter species was ranked as the fourth most frequent pathogen among gram-negative bacilli following E. coli $(n=536), K$. pneumoniae $(n=196)$, and Aeromonas species $(n=62)$.

The 32 Enterobacter SBP patients (cases) were ageand sex-matched with 128 control subjects who had $E$. coli SBP. The demographic characteristics of the patients are summarized in Table 1 . In both groups, $81.3 \%$ were male and the mean age was 55 years. The Enterobacter SBP patients had a significantly higher percentage of definite SBP cases $(81.2 \%$ vs. $60.9 \%, P=0.03)$, hospitalacquired infections $(62.5 \%$ vs. $23.4 \%, P<0.001)$, and concomitant hepatocellular carcinoma (HCC) $(65.6 \%$ vs. $37.5 \%, P=0.004)$, whereas they had a significantly lower proportion of cases with Child-Pugh score C $(65.6 \%$ vs. $86.7 \%, P=0.006)$ and median value of MELD score (19 vs. $23, P=0.03)$. Antecedent endoscopic interventions had more frequently been performed in the Enterobacter SBP group ( $25.0 \%$ vs. $5.5 \%, P=0.001$ ), and the median period between endoscopy and the diagnosis of Enterobacter SBP was 22 days (range, 2 - 30 days). Of the eight Enterobacter cases involving prior endoscopic intervention, three occurred within 5 days of the endoscopy whereas the remaining five developed more than 3 weeks 
after the procedure. Anticancer chemotherapy $(9.4 \%$ vs. $0 \%, P=0.007)$ and prior exposure to antimicrobial agents (59.4 \% vs. $28.1 \%, P=0.001)$ within 30 days were more common in the cases. Multivariate analysis showed that hospital acquisition (OR, 3.19; $95 \% \mathrm{CI}, 1.25-8.14 ; P=$ 0.02 ) and prior endoscopic intervention (OR, 3.57; $95 \%$ CI, $1.02-12.44 ; \quad P=0.046)$ were independent factors favoring Enterobacter SBP rather than E. coli SBP. Results of univariate and multivariate analysis are shown in Additional file 1: Table S1.

\section{Clinical features and laboratory findings}

Table 2 compares the clinical features of SBP and the laboratory findings between the groups. Abdominal pain and fever were the most common initial manifestations in both groups. Upper gastrointestinal bleeding episodes were significantly more frequent in the Enterobacter SBP than in the E. coli SBP group $(28.1 \%$ vs. $9.4 \%, P=$ 0.005). Concomitant bacteremia was less common in cases than in controls $(34.4 \%$ vs. $68.0 \%, P=0.001)$. The laboratory findings in the Enterobacter SBP group vs. the E. coli group revealed a trend towards higher levels of systemic inflammatory markers such as serum white blood cell count (median, 8,050 cells/ $\mu \mathrm{L}$ vs. 6,450 cells/ $\mu \mathrm{L}, P=0.12$ ) and serum $\mathrm{C}$-reactive protein (median, $6.41 \mathrm{mg} / \mathrm{dL}$ vs. $3.06 \mathrm{mg} / \mathrm{dL}, P=0.06$ ), but the differences were not statistically significant.

\section{Antimicrobial susceptibility}

Susceptibilities of 31 and 125 clinical isolates of Enterobacter species and E. coli, respectively, to a range of antimicrobials are shown in Table 3. Enterobacter species from 22 patients $(71.0 \%)$ and E. coli from 106 patients $(84.8 \%)$ were susceptible to third-generation cephalosporins and susceptibility of Enterobacter SBP group tended to be lower than $E$. coli SBP group $(P=0.07)$. Rates of susceptibility to ciprofloxacin $(80.6 \%$ vs. $60.8 \%, P=$ $0.038)$ and trimethoprim-sulfamethoxazole $(87.1 \%$ vs. $59.2 \%, P=0.003)$ were higher in the case group, whereas piperacillin/tazobactam susceptibility was lower in the case group ( $74.2 \%$ vs. $92.8 \%, P=0.003)$. Of the 31 Enterobacter SBP patients for whom there were antimicrobial susceptibility test results, $19(61.3 \%)$ had been exposed to antecedent antimicrobials. Of those 19 patients, 16 had histories of exposure to 3rd-generation cephalosporins. The frequency of resistance to $3^{\text {rd }}$-generation cephalosporins of the Enterobacter isolates from patients with prior antimicrobial exposure was twice as high as that in the unexposed group, but the difference was not statistically significant $(36.8 \%[7 / 19]$ vs. $16.7 \%[2 / 12], P=0.42)$.

\section{Empirical treatment, outcomes}

Antimicrobial treatments and outcomes are summarized in Table 4. The majority of patients in both groups were treated empirically with cefotaxime (84.4 and $90.6 \%$, respectively). The percentages of appropriateness of the initial therapy were similar between the groups $(87.1 \%$ vs. $87.2 \%, P=1.00)$. Treatment outcomes including response to initial therapy $(81.3 \%$ vs. $81.2 \%, P=0.995)$ and 30 -day mortality (37.5 \% vs. $28.9 \%, P=0.35$ ) were not significantly different between groups. The lengths of hospital stay were similar between the case group (median, 20 days; IQR, $11-31$ days) and control group (16 days; IQR, $10-26$ days, $P=0.28$ ). The duration of antimicrobial use was significantly longer in the cases (median, 15 days; IQR, $11-25$ days) than in the controls (median, 13 days; IQR, $8-16$ days, $P=0.02$ ). There were 2 cases of recurrent SBP in Enterobacter SBP group. Among 2 Enterobacter isolates from recurrent episode, one was susceptible and the other was resistant to $3^{\text {rd }}$ generation cephalosporins. Antimicrobial susceptibility pattern from those 2 Enterobacter isolates were not changed at recurrent episodes.

\section{Emergence of resistance}

A large subgroup of patients was initially treated with third-generation cephalosporins: 27 cases received cefotaxime, and 117 controls received either cefotaxime or ceftazidime. Of these, 23 patients in the Enterobacter SBP group and 98 in the E. coli SBP group were susceptible to empirical antimicrobial treatment. Emergence of resistance during antimicrobial therapy was identified in one case $(4.3 \% ; 1$ of 23$)$ and in no controls $(P=0.19)$.

\section{Discussion}

In this retrospective, case-matched study, we investigated the characteristics and outcomes of the Enterobacter SBP. Compared with E. coli SBP, patients in the Enterobacter SBP group had less severe liver diseases and more commonly had concomitant HCC; they were more likely to have had prior endoscopic interventions and to present with upper gastrointestinal bleeding. However, response to empirical therapy and 30-day mortality rate were not significantly different between the groups. Emergence of resistance to third-generation cephalosporins was infrequent in both groups.

Enterobacter species are usually regarded as nosocomial pathogens $[1,19]$. According to different studies, $56-100 \%$ of Enterobacter infections were acquired institutionally [10, 20-23]. Among the 32 Enterobacter SBP patients in our study, 20 cases $(62.5 \%)$ had hospital-acquired infections; this proportion is within the range of prior reports. Prior antimicrobial usage was also more common in the Enterobacter SBP group than in the E. coli group. This finding is consistent with previous studies in which prior use of antimicrobial agents was a crucial risk factor for Enterobacter infection $[1,10,24-26]$. Interestingly, about two-thirds of the 
Enterobacter SBP group had concomitant HCC; this proportion is significantly higher than in the E. coli group, whereas the patients in the Enterobacter SBP group had less severe hepatic impairment than the case group. We presume that the treatment of $\mathrm{HCC}$, or $\mathrm{HCC}$ per se, may contribute to the development of SBP by increasing the probability of hospitalization or antimicrobial exposure.

We found that prior endoscopic interventions were more frequently performed in the Enterobacter group than in the E. coli group. The rate of transient bacteremia after endoscopic intervention has been reported to be substantial, within the range of $6-53 \%$ [27-32]. Bacteremia could lead to subsequent SBP in patients with cirrhotic ascites. A few studies showed that endoscopic interventions for varix control, including endoscopic variceal ligation and endoscopic sclerotherapy, could be complicated by SBP in $0.5-3.0 \%$ of cases [32-34]. The interval between the endoscopic intervention and a diagnosis of SBP in those studies was about 2-4 days. Of the eight Enterobacter SBP patients in our study who received prior endoscopic intervention, three cases were diagnosed with SBP within 5 days and the remaining five cases developed more than 3 weeks after the procedure. Therefore, only the three cases with a short interval between endoscopy and SBP development seem to be directly related to the endoscopic procedure. Intriguingly, we found that upper gastrointestinal bleeding occurred more frequently in the Enterobacter group, despite the higher frequency of severe liver disease in the E. coli group. Of the nine Enterobacter SBP patients with bleeding, seven had HCC, all in an unresectable state. Three of these HCC cases were complicated by portal vein tumor thrombosis and one case by direct duodenal wall invasion of the cancer. Portal vein thrombosis has been considered a risk factor for variceal bleeding [35-37]. We conjecture that more frequent bleeding episodes in the Enterobacter group could be explained in part by complications of advanced HCC.

Although susceptibility to third-generation cephalosporins tended to be lower in the Enterobacter group, the percentages of appropriateness of antimicrobial therapy, the initial responses to empirical therapy, and the 30-day mortality rates were comparable between groups. The development of resistance during third-generation cephalosporin therapy has been a great concern to clinicians. However, the frequency of resistance arising during third-generation cephalosporin therapy in our study $(4.3 \%, 1 / 23)$ was much lower than those in previous studies regarding Enterobacter bloodstream infections $(8.3-19.6 \%)[10,11]$. Cefotaxime, the most widely used initial empiric therapy for SBP in cirrhosis, can penetrate into ascites rapidly and achieve a higher drug concentration in ascites than in blood [38-41].
This pharmacokinetic distinctiveness of the antimicrobial agent could explain the lower rate of emergence of resistance in our study population. Since Enterobacter SBP accounted for only $2.7 \%$ of the whole SBP cohort and the treatment outcomes of the Enterobacter SBP patients were comparable to those of the E. coli SBP, it seems likely that a change of treatment strategy to target Enterobacter species is unnecessary.

There are several limitations of this study. First, we conducted a retrospective, case-matched study in a single tertiary center with a limited sample size, which does not permit generalization of our results. Although age and sex were matched in the control group, unexpected confounding factors may have been overlooked. Second, since the isolates were not available, the emergence of resistance during therapy could not be confirmed by molecular methods such as pulsed-field gel electrophoresis. Finally, $E$. cloacae and E. aerogenes were analyzed together as a group. The possible differences of antimicrobial resistance pattern and mortality between the two species may have affected antimicrobial susceptibility profile and outcomes in our study [42].

\section{Conclusions}

Our study shows clinical features and treatment outcomes of spontaneous bacterial peritonitis caused by Enterobacter species. Compared with E. coli SBP, Enterobacter SBP was more commonly associated with underlying hepatocellular carcinoma and upper gastrointestinal bleeding. Clinical outcomes associated with SBP were comparable between the groups. Development of resistance during third-generation cephalosporin therapy was infrequently identified.

\section{Additional file}

Additional file 1: Table S1 Univariate and multivariate factors associated with Enterobacter spontaneous bacterial peritonitis vs. E. coli spontaneous bacterial peritonitis. (DOCX $21 \mathrm{~kb}$ )

Abbreviations

SBP, spontaneous bacterial peritonitis; MELD, model for end-stage liver disease; PMN, polymorphonuclear cell; HCC, hepatocellular carcinoma; IQR, interquartile range; ERCP, endoscopic retrograde cholangiopancreatography; WBC, white blood cells; ICU, intensive care unit

\section{Acknowledgements \\ None.}

Funding

This study was supported by a grant from the Asan Institute of Life Sciences (Grant 2010-0079).

Availability of data and materials

All the data supporting our findings are contained within this work.

Authors' contributions

SHC, SOL and SHK conceived, initiated and designed the study and coordinated drafting the manuscript. SB and SHC carried out data analysis, 
and wrote the manuscript. MCK, TK and YPJ were responsible for the data collection, reviewing medical charts and assisted in performing statistical analyses. YSL participated in interpretation and evaluation of treatment responses and assisted in data analysis. YSK and JHW supervised the study design, reviewed and commented the manuscript, and approved the final draft. MNK and SH supervised and check the issues of microbiologic and laboratory data. All authors read and approved the final manuscript.

\section{Competing interests}

The authors declare that they have no competing interest.

\section{Ethics approval and consent to participate}

The institutional review board of the Asan Medical Center gave approval to conduct this research with a waiver of informed consent.

\section{Author details}

${ }^{1}$ Department of Infectious Diseases, Asan Medical Center, University of Ulsan College of Medicine, Seoul, Republic of Korea. ${ }^{2}$ Department of Gastroenterology, Asan Medical Center, University of Ulsan College of Medicine, Seoul, Republic of Korea. ${ }^{3}$ Department of Laboratory Medicine, Asan Medical Center, University of Ulsan College of Medicine, Seoul, Republic of Korea.

Received: 4 October 2015 Accepted: 26 May 2016 Published online: 07 June 2016

\section{References}

1. Sanders Jr WE, Sanders CC. Enterobacter spp.: pathogens poised to flourish at the turn of the century. Clin Microbiol Rev. 1997;10(2):220-41.

2. Wisplinghoff $H$, Bischoff $T$, Tallent $S M$, Seifert $H$, Wenzel RP, Edmond MB Nosocomial bloodstream infections in US hospitals: analysis of 24,179 cases from a prospective nationwide surveillance study. Clin Infect Dis. 2004:39(3):309-17. doi:10.1086/421946.

3. Diekema DJ, Pfaller MA, Jones RN, Doern GV, Kugler KC, Beach ML, et al. Trends in antimicrobial susceptibility of bacterial pathogens isolated from patients with bloodstream infections in the USA, Canada and Latin America. SENTRY Participants Group. Int J Antimicrob Agents. 2000;13(4):257-71.

4. Adam HJ, DeCorby M, Rennie R, Karlowsky JA, Hoban DJ, Zhanel GG. Prevalence of antimicrobial resistant pathogens from blood cultures from Canadian hospitals: results of the CANWARD 2007-2009 study. Diagn Microbiol Infect Dis. 2011:69(3):307-13. doi:10.1016/j.diagmicrobio.2010.10.026.

5. Ibrahim EH, Sherman G, Ward S, Fraser VJ, Kollef MH. The influence of inadequate antimicrobial treatment of bloodstream infections on patient outcomes in the ICU setting. Chest. 2000;118(1):146-55.

6. Diekema DJ, Pfaller MA, Jones RN, Doern GV, Winokur PL, Gales AC, et al. Survey of bloodstream infections due to gram-negative bacilli: frequency of occurrence and antimicrobial susceptibility of isolates collected in the United States, Canada, and Latin America for the SENTRY Antimicrobial Surveillance Program, 1997. Clin Infect Dis. 1999; 29(3):595-607.

7. Sader HS, Biedenbach DJ, Jones RN. Global patterns of susceptibility for 21 commonly utilized antimicrobial agents tested against 48,440 Enterobacteriaceae in the SENTRY Antimicrobial Surveillance Program (1997-2001). Diagn Microbiol Infect Dis. 2003;47(1):361-4

8. Schmidtke AJ, Hanson ND. Model system to evaluate the effect of ampD mutations on AmpC-mediated beta-lactam resistance. Antimicrob Agents Chemother. 2006;50(6):2030-7. doi:10.1128/aac.01458-05.

9. Jacoby GA. AmpC beta-lactamases. Clin Microbiol Rev. 2009;22(1):161-82. doi:10.1128/cmr.00036-08. Table of Contents.

10. Chow JW, Fine MJ, Shlaes DM, Quinn JP, Hooper DC, Johnson MP, et al. Enterobacter bacteremia: clinical features and emergence of antibiotic resistance during therapy. Ann Intern Med. 1991;115(8):585-90.

11. Choi SH, Lee JE, Park SJ, Choi SH, Lee SO, Jeong JY, et al. Emergence of antibiotic resistance during therapy for infections caused by Enterobacteriaceae producing AmpC beta-lactamase: implications for antibiotic use. Antimicrob Agents Chemother. 2008:52(3):995-1000. doi:10.1128/aac.01083-07.
12. Fernandez J, Navasa M, Gomez J, Colmenero J, Vila J, Arroyo V, et al. Bacterial infections in cirrhosis: epidemiological changes with invasive procedures and norfloxacin prophylaxis. Hepatology. 2002;35(1):140-8. doi:10.1053/jhep.2002.30082.

13. Singh N, Wagener MM, Gayowski T. Changing epidemiology and predictors of mortality in patients with spontaneous bacterial peritonitis at a liver transplant unit. Clin Microbiol Infect. 2003;9(6):531-7.

14. Park $\mathrm{YH}$, Lee HC, Song HG, Jung S, Ryu SH, Shin JW, et al. Recent increase in antibiotic-resistant microorganisms in patients with spontaneous bacterial peritonitis adversely affects the clinical outcome in Korea. J Gastroenterol Hepatol. 2003;18(8):927-33.

15. Cheong HS, Kang Cl, Lee JA, Moon SY, Joung MK, Chung DR, et al. Clinical significance and outcome of nosocomial acquisition of spontaneous bacterial peritonitis in patients with liver cirrhosis. Clin Infect Dis. 2009;48(9): 1230-6. doi:10.1086/597585.

16. EASL clinical practice guidelines on the management of ascites, spontaneous bacterial peritonitis, and hepatorenal syndrome in cirrhosis. J Hepatol. 2010;53(3):397-417. doi:10.1016/j.jhep.2010.05.004.

17. Rimola A, Garcia-Tsao G, Navasa M, Piddock L, Planas R, Bernard B, et al. Diagnosis, treatment and prophylaxis of spontaneous bacterial peritonitis: a consensus document. International Ascites Club. J Hepatol. 2000;32(1):142-53.

18. Clinical and Laboratory Standards Institute. Performance standards for antimicrobial susceptibility testing. 19th informational supplement M100S19. Wayne, PA. CLSI 2009

19. John Jr JF, Sharbaugh RJ, Bannister ER. Enterobacter cloacae: bacteremia, epidemiology, and antibiotic resistance. Rev Infect Dis. 1982;4(1):13-28.

20. Andresen J, Asmar BI, Dajani AS. Increasing Enterobacter bacteremia in pediatric patients. Pediatr Infect Dis J. 1994:13(9):787-92.

21. Matsaniotis NS, Syriopoulou VP, Theodoridou MC, Tzanetou KG, Mostrou GI. Enterobacter sepsis in infants and children due to contaminated intravenous fluids. Infect Control. 1984;5(10):471-7.

22. Hennigs JK, Baumann HJ, Schmiedel S, Tennstedt P, Sobottka I, Bokemeyer C, et al. Characterization of Enterobacter cloacae pneumonia: a single-center retrospective analysis. Lung. 2011;189(6):475-83. doi:10.1007/s00408-011-9323-2.

23. Huang CR, Lu CH, Chang WN. Adult Enterobacter meningitis: a high incidence of coinfection with other pathogens and frequent association with neurosurgical procedures. Infection. 2001;29(2):75-9.

24. Chen HL, Lu JH, Wang HH, Chen SJ, Chen CJ, Wu KG, et al. Clinical analysis of Enterobacter bacteremia in pediatric patients: a 10-year study. J Microbiol Immunol Infect. 2014;47(5):381-6. doi:10.1016/j.jmii.2013.03.016.

25. Chow JW, Yu VL, Shlaes DM. Epidemiologic perspectives on Enterobacter for the infection control professional. Am J Infect Control. 1994;22(4):195-201.

26. al Ansari N, McNamara EB, Cunney RJ, Flynn MA, Smyth EG. Experience with Enterobacter bacteraemia in a Dublin teaching hospital. J Hosp Infect. 1994;27(1):69-72.

27. Sauter G, Grabein B, Huber G, Mannes GA, Ruckdeschel G, Sauerbruch T. Antibiotic prophylaxis of infectious complications with endoscopic retrograde cholangiopancreatography. A randomized controlled study. Endoscopy. 1990;22(4):164-7. doi:10.1055/s-2007-1012830.

28. Kullman E, Borch K, Lindstrom E, Ansehn S, Ihse I, Anderberg B. Bacteremia following diagnostic and therapeutic ERCP. Gastrointest Endosc. 1992:38(4):444-9.

29. Lo GH, Lai KH, Shen MT, Chang CF. A comparison of the incidence of transient bacteremia and infectious sequelae after sclerotherapy and rubber band ligation of bleeding esophageal varices. Gastrointest Endosc. 1994;40(6):675-9.

30. Mollison LC, Desmond PV, Stockman KA, Andrew JH, Watson K, Shaw G, et al. A prospective study of septic complications of endoscopic retrograde cholangiopancreatography. J Gastroenterol Hepatol. 1994;9(1):55-9.

31. Niederau C, Pohlmann U, Lubke H, Thomas L. Prophylactic antibiotic treatment in therapeutic or complicated diagnostic ERCP: results of a randomized controlled clinical study. Gastrointest Endosc. 1994;40(5):533-7.

32. Lin OS, Wu SS, Chen YY, Soon MS. Bacterial peritonitis after elective endoscopic variceal ligation: a prospective study. Am J Gastroenterol. 2000;95(1):214-7. doi:10.1111/j.1572-0241.2000.01687.x.

33. Bac DJ, de Marie S, Siersema PD, Snobl J, van Buuren HR. Post-sclerotherapy bacterial peritonitis: a complication of sclerotherapy or of variceal bleeding? Am J Gastroenterol. 1994;89(6):859-62.

34. Schembre D, Bjorkman DJ. Post-sclerotherapy bacterial peritonitis. Am J Gastroenterol. 1991;86(4):481-6. 
35. Kadouchi K, Higuchi K, Shiba M, Okazaki H, Yamamori K, Sasaki E, et al. What are the risk factors for aggravation of esophageal varices in patients with hepatocellular carcinoma? J Gastroenterol Hepatol. 2007;22(2):240-6. doi:10.1111/j.1440-1746.2006.04418.x.

36. Lang BH, Poon RT, Fan ST, Wong J. Outcomes of patients with hepatocellular carcinoma presenting with variceal bleeding. Am J Gastroenterol. 2004;99(11):2158-65. doi:10.1111/j.1572-0241.2004.40336.x.

37. Chen $\mathrm{CH}$, Sheu JC, Huang GT, Lee HS, Yang PM, Wong JM, et al. Characteristics of hepatocellular carcinoma presenting with variceal bleeding. J Gastroenterol Hepatol. 1998;13(2):170-4.

38. Dalmau D, Layrargues GP, Fenyves D, Willems B, Turgeon F, Turgeon P. Cefotaxime, desacetyl-cefotaxime, and bactericidal activity in spontaneous bacterial peritonitis. J Infect Dis. 1999;180(5):1597-602. doi:10.1086/315053.

39. Runyon BA, Akriviadis EA, Sattler FR, Cohen J. Ascitic fluid and serum cefotaxime and desacetyl cefotaxime levels in patients treated for bacterial peritonitis. Dig Dis Sci. 1991;36(12):1782-6.

40. Ko RJ, Sattler FR, Nichols S, Akriviadis E, Runyon B, Appleman M, et al. Pharmacokinetics of cefotaxime and desacetylcefotaxime in patients with liver disease. Antimicrob Agents Chemother. 1991;35(7):1376-80.

41. Hary L, Andrejak M, Leleu S, Orfila J, Capron JP. The pharmacokinetics of ceftriaxone and cefotaxime in cirrhotic patients with ascites. Eur J Clin Pharmacol. 1989:36(6):613-6.

42. Song EH, Park KH, Jang EY, Lee EJ, Chong YP, Cho OH, et al. Comparison of the clinical and microbiologic characteristics of patients with Enterobacter cloacae and Enterobacter aerogenes bacteremia: a prospective observation study. Diagn Microbiol Infect Dis. 2010;66(4):436-40.

\section{Submit your next manuscript to BioMed Central} and we will help you at every step:

- We accept pre-submission inquiries

- Our selector tool helps you to find the most relevant journal

- We provide round the clock customer support

- Convenient online submission

- Thorough peer review

- Inclusion in PubMed and all major indexing services

- Maximum visibility for your research

Submit your manuscript at www.biomedcentral.com/submit 